Supporting Information

\title{
Dichlorocarbene Addition to Cyclopropenes: A Computational Study
}

\author{
Dina C. Merrer ${ }^{\dagger *}$ and Paul R. Rablen ${ }^{\ddagger}$ \\ ${ }^{\dagger}$ Department of Chemistry, Barnard College, New York, NY 10027, and \\ ${ }^{\ddagger}$ Department of Chemistry and Biochemistry, Swarthmore College, Swarthmore, PA 19081
}

\begin{tabular}{lc}
\hline Contents & Pages \\
\hline Gas phase stationary point geometries at B3LYP/6-31G* - Path A & S1-S4 \\
Gas phase stationary point geometries at B3LYP/6-31G* - Path B & S4-S8 \\
Stationary point geometries in MeCN (SCRF = PCM) at B3LYP/6-31G* - Path A & S8-S10 \\
\hline
\end{tabular}

Absolute energies are given in hartrees and are uncorrected. The imaginary frequency of each transition state is provided.

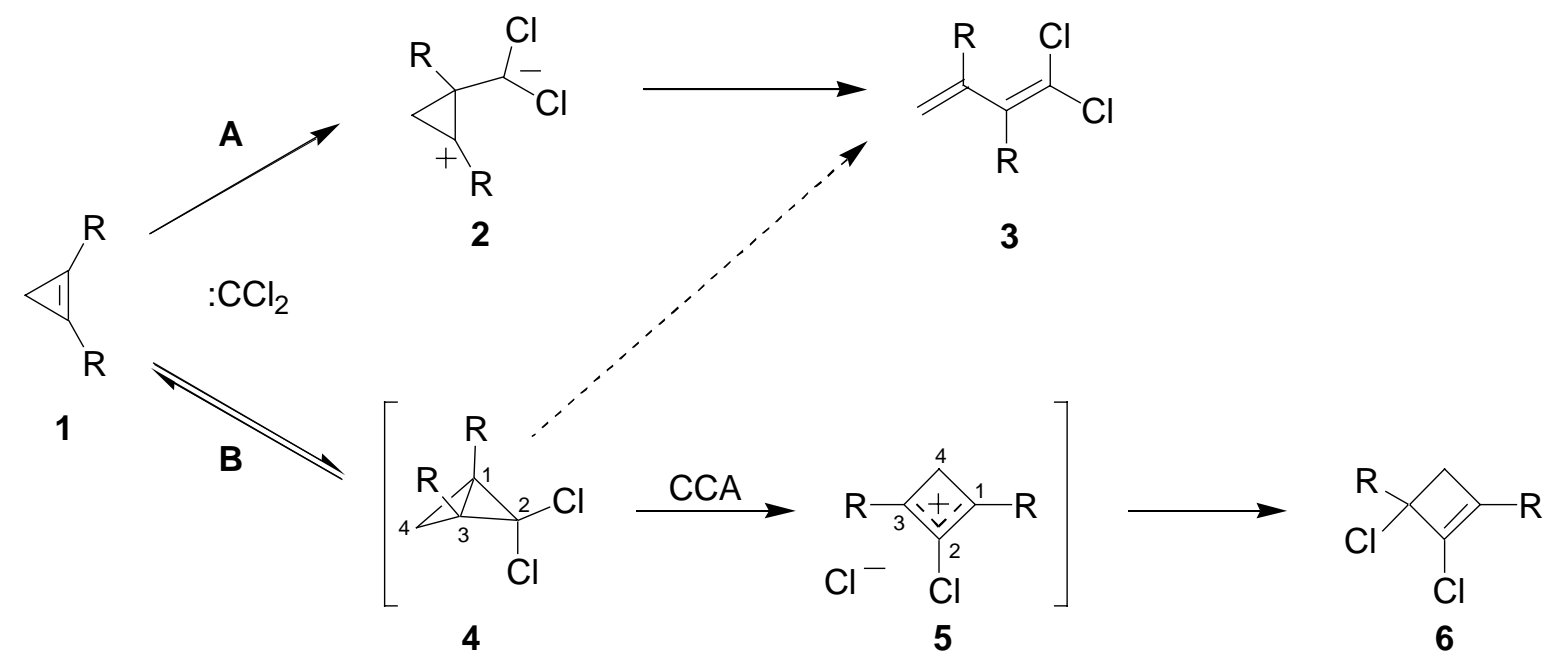

$\mathbf{a}, \mathrm{R}=\mathrm{H} ; \mathbf{b}, \mathrm{R}=\mathrm{CH}_{3} ; \mathbf{c}, \mathrm{R}=\mathrm{CH}=\mathrm{CH}_{2} ; \mathbf{d}, \mathrm{R}=\mathrm{Ph} ; \mathbf{e}, \mathrm{R}=\left(p-\mathrm{OCH}_{3}\right) \mathrm{C}_{6} \mathrm{H}_{4} ; \mathbf{f}, \mathrm{R}=\left(p-\mathrm{CF}_{3}\right) \mathrm{C}_{6} \mathrm{H}_{4}$

Gas phase stationary point geometries at B3LYP/6-31G* - Path A

Cyclopropene $\mathbf{1}+: \mathrm{CCl}_{2}$

$\mathbf{R}=\mathbf{H}(r=3.4 \AA)$

Abs. $\mathrm{E}=-1075.00425703$

C, $2.409179,-0.971866,-0.395177$

C, 2.130157, $-0.424781,0.747968$

$\mathrm{H}, 1.751012,-0.505225,1.754972$

$\mathrm{H}, 2.423504,-1.842664,-1.033798$

C, 2.747483, 0.491250, -0.279959

C, $-0.997635,0.025804,0.776706$

$\mathrm{H}, 3.791449,0.803829,-0.175732$

$\mathrm{H}, 2.126430,1.237731,-0.786869$
$\mathbf{R}=\mathbf{C H}_{\mathbf{3}}(r=3.3 \AA)$

Abs. $\mathrm{E}=-1153.65507903$

C, $-1.761670,0.592938,-0.468174$

C, $-1.930398,-0.414340,0.342529$

C, $-2.082975,-0.987176,1.699363$

C, $-1.560333,2.035677,-0.730220$

C, $-1.972841,-0.745433,-1.129146$

C, $1.323597,-0.388840,0.721282$

$\mathrm{H},-1.131535,-1.269756,-1.600510$

$\mathrm{H},-2.932162,-0.971342,-1.612388$ 
$\mathrm{Cl},-1.815534,-1.206876,-0.168587$

$\mathrm{Cl},-0.997849,1.535340,-0.117049$

$\mathbf{R}=\mathbf{C H}=\mathbf{C H}_{2}(r=3.4 \AA)$

Abs. $\mathrm{E}=-1229.82076262$

C, $1.675277,0.579903,0.097126$

C, $1.733528,-0.679070,0.486764$

C, $1.726813,-2.089863,0.211622$

C, $1.554864,1.576871,-0.923075$

C, $1.869175,0.355325,1.574763$

C, $-1.657457,-0.070491,0.799357$

H, $1.035525,0.526633,2.261394$

$\mathrm{H}, 2.851969,0.543522,2.021254$

Cl, $-2.170815,-1.440113,-0.172838$

$\mathrm{Cl},-2.169068,1.416570,0.018515$

$\mathrm{H}, 1.827890,-2.745920,1.074240$

C, $1.601126,-2.626235,-1.015152$

H, 1.599477, $-3.701911,-1.162736$

H, 1.498669, $-2.007265,-1.902543$

$\mathrm{H}, 1.453200,1.224479,-1.949160$

C, 1.560676, 2.896833, -0.666021

H, 1.468206, 3.630682, -1.460649

H, 1.659060, 3.270383, 0.349383
$\mathrm{Cl}, 1.984810,1.231045,0.509057$

$\mathrm{Cl}, 1.712880,-1.356178,-0.692835$

$\mathrm{H},-1.310199,-1.741524,1.887772$

H, $-3.057094,-1.483324,1.799588$

$\mathrm{H},-2.002213,-0.216071,2.472539$

$\mathrm{H},-0.628870,2.205420,-1.286802$

$\mathrm{H},-1.515401,2.613562,0.198886$

H, $-2.375533,2.433351,-1.348666$

$\mathbf{R}=\mathbf{P h}(r=2.5 \AA)$

Abs. $E=-1537.14005348$

C, $-0.733507,0.029085,-1.000593$

C, $0.584870,-0.154259,-1.073481$

C, $1.828447,-0.681775,-0.590013$

C, $-2.044860,-0.405412,-0.561119$

C, $-0.035829,0.610786,-2.203096$

C, $-0.419064,1.880951,0.497175$

$\mathrm{H}, 0.097563,1.692398,-2.282142$

H, $-0.149678,0.109387,-3.170408$

$\mathrm{Cl}, 0.992568,2.915895,0.107202$

$\mathrm{Cl},-0.123572,1.137057,2.093364$

C, $1.861621,-1.439752,0.585995$

C, $3.017345,-0.428742,-1.283643$

C, 3.076577, - $1.944807,1.062888$

C, $4.230563,-0.940400,-0.809368$

C, $4.263519,-1.697900,0.363725$

$\mathrm{H}, 0.940956,-1.630040,1.129605$

H, 2.994199, 0.166211, -2.191849

H, 3.093920, $-2.530815,1.977021$

H, 5.148244, $-0.741948,-1.355099$

H, 5.201612, $-2.096410,0.738581$

C, $-2.167074,-1.445756,0.366983$

C, $-3.196431,0.196883,-1.080493$

C, $-3.433859,-1.880387,0.773156$

C, $-4.462444,-0.237173,-0.671309$

C, $-4.584405,-1.275031,0.255069$

$\mathrm{H},-1.274661,-1.923678,0.760091$

$\mathrm{H},-3.103123,1.008263,-1.796245$

$\mathrm{H},-3.520085,-2.690882,1.490796$

$\mathrm{H},-5.351032,0.237130,-1.077257$

H, $-5.563659,-1.617661,0.576022$

Cyclopropene $1+: \mathrm{CCl}_{2}-$ Transition state for stepwise addition

$\mathbf{R}=\mathbf{H}(r=2.5 \AA)$

Abs. $\mathrm{E}=-1075.00349154$

C, $-1.720594,-0.675830,0.783142$

C, $-1.585324,-0.891788,-0.504573$

$\mathrm{H},-1.334587,-1.574337,-1.299536$

$\mathrm{H},-1.481766,-0.955796,1.798233$

C, $-2.490997,0.247326,-0.108035$

C, $0.551922,0.037645,-0.705530$

$\mathrm{H},-3.576175,0.126922,-0.184031$

H, $-2.155697,1.276724,-0.262214$

$\mathrm{Cl}, 1.664155,-1.103968,0.092715$

$\mathrm{Cl}, 0.689856,1.622931,0.093022$
$\mathbf{R}=\mathbf{C H}_{3}$

All structures with $r<3.4 \AA$ were lower in energy than the starting structure $(r=3.4 \AA)$. 
Imag. freq. $=142.2$

$\mathbf{R}=\mathbf{C H}=\mathbf{C H}_{\mathbf{2}}(r=2.5 \AA)$

Abs. $\mathrm{E}=-1229.82046304$

C, $-0.161774,1.373509,-0.015139$

C, $-1.109321,0.706153,-0.672301$

C, $-2.396289,0.050161,-0.699055$

C, $0.515433,1.731274,1.187726$

C, $-0.191276,1.568373,-1.501752$

C, $0.229362,-1.293273,-0.585717$

$\mathrm{H}, 0.564683,1.101325,-2.137844$

$\mathrm{H},-0.572475,2.516486,-1.895368$

Cl, 0.013558, -1.920791, 1.072308

$\mathrm{Cl}, 1.973331,-0.999135,-0.843182$

H, $-2.701148,-0.354208,-1.661196$

C, $-3.197837,-0.064078,0.369170$

$\mathrm{H},-4.161012,-0.560728 \quad 0.299896$

$\mathrm{H},-2.918655,0.336695,1.339585$

H, $0.130570,1.321190 \quad 2.119694$

C, 1.605240, 2.522072, 1.192820

$\mathrm{H}, 2.116488,2.778103,2.115450$

H, 2.003199, 2.934746, 0.270135

Imag. freq. $=106.0$

\section{1,3-Dihalobutadiene 3}

$\mathbf{R}=\mathbf{H}$

Abs. $\mathrm{E}=-1075.17879836$

C, $-1.989604,-1.020220,0.000001$

C, $-0.530303,-1.107631,-0.000003$

$\mathrm{H},-0.137422,-2.121348,-0.000001$

H, -2.463965, -2.000503, 0.000005

C, $-2.798261,0.051831,0.000001$

C, $0.420017,-0.157331,-0.000006$

$\mathrm{H},-2.440775,1.073539,-0.000001$

$\mathrm{H},-3.875306,-0.087993,0.000005$

$\mathrm{Cl}, 2.116471,-0.583620,0.000002$

$\mathrm{Cl}, 0.136845,1.556350,0.000000$

\section{$\mathbf{R}=\mathbf{C H}=\mathbf{C H}_{2}$}

Abs. $\mathrm{E}=-1229.95916158$

C, 1.463543, 0.133557, 0.368606

C, $0.036453,0.529708,0.082091$

C, $1.960288,0.241541,1.608704$

C, $-0.919066,-0.415657,0.000400$

$\mathrm{H}, 1.356118,0.607727,2.433405$

H, 2.980900, $-0.054575,1.833600$

$\mathrm{Cl},-2.584569,-0.122923,-0.428289$

$\mathrm{Cl},-0.586224,-2.109327,0.283449$

C, $-0.147526,1.974615,-0.156775$

\section{$\mathbf{R}=\mathbf{P h}$}

All structures with $r<2.5 \AA$ were lower in energy than the starting structure $(r=2.5 \AA)$.

$\mathbf{R}=\mathbf{C H}_{3}$

Abs. $\mathrm{E}=-1153.80536316$

C, $-1.727622,0.084480,0.094922$

C, $-0.355992,0.692598,0.044513$

C, $-2.358068,-0.077013,1.261547$

C, 0.739540, $-0.077815,0.022218$

$\mathrm{H},-1.887954,0.175039,2.208213$

H, $-3.366313,-0.481202,1.307462$

Cl, 2.376078, 0.544039, -0.054273

$\mathrm{Cl}, 0.680771,-1.82578,0.065980$

C, $-0.314917,2.200813,-0.004752$

$\mathrm{H},-0.705924,2.613747,0.933701$

$\mathrm{H}, 0.690000,2.592913,-0.167718$

$\mathrm{H},-0.967412,2.568085,-0.806751$

C, $-2.351079,-0.261095,-1.236826$

$\mathrm{H},-2.383421,0.612734,-1.900913$

H, $-1.763983,-1.030820,-1.752472$

H, $-3.372599,-0.632605,-1.110263$

$\mathbf{R}=\mathbf{P h}$

Abs. $\mathrm{E}=-1537.27623279$

C, $-0.724543,-0.424268,0.343229$

C, 0.560291, $-0.919047,0.969326$

C, $1.830125,-0.610512,0.253730$

C, $-0.820549,1.068718,0.240962$

C, $0.525750,-1.562345,2.146790$

C, $-1.743169,-1.225880,-0.081133$

$\mathrm{H}, 1.432733,-1.922084,2.624320$

H, $-0.411384,-1.763382,2.655819$

$\mathrm{Cl},-1.645800,-2.971869,-0.087930$ 
C, $2.260051,-0.304115,-0.797832$

H, 0.715077, 2.443271, -0.629672

C, $-1.177898,2.749374,0.202913$

$\mathrm{H},-1.161171,3.814938,-0.007237$

$\mathrm{H},-2.051057,2.366929,0.719656$

$\mathrm{H}, 1.723338,-0.885551,-1.546550$

C, 3.548857, $-0.006683,-1.001209$

$\mathrm{H}, 4.081818,-0.374284,-1.873343$

H, 4.110242, 0.615758, -0.308959
$\mathrm{Cl},-3.282389,-0.635446,-0.651645$

C, $1.885256,-0.661807,-1.142591$

C, $2.985837,-0.287685,0.971710$

C, 3.087964, $-0.414564,-1.811732$

C, $4.187615,-0.038977,0.301442$

C, 4.245796, $-0.103249,-1.092086$

H, 0.992556, $-0.912580,-1.708557$

H, 2.943140, $-0.206965,2.054198$

H, 3.121996, - $0.466120,-2.896306$

H, 5.078234, 0.216839, 0.868429

H, 5.173562, 0.093013, -1.622039

C, $-1.045905,1.679597,-0.996546$

C, $-0.627771,1.868697,1.371562$

C, $-1.095730,3.073387,-1.097793$

C, $-0.689307,3.261998,1.270032$

C, $-0.922286,3.870899,0.037670$

$\mathrm{H},-1.175061,1.067269,-1.884515$

$\mathrm{H},-0.432620,1.404020,2.333907$

$\mathrm{H},-1.266229,3.537954,-2.064864$

$\mathrm{H},-0.549663,3.873880,2.156722$

H, $-0.964315,4.952727,-0.051777$

\section{Gas phase stationary point geometries at B3LYP/6-31G* - Path B}

Transition state connecting $\mathbf{1}+: \mathrm{CCl}_{2}$ to bicyclobutane $\mathbf{4}$

\section{$\mathbf{R}=\mathbf{H}$}

Abs. $\mathrm{E}=-1075.0034884$

C, $-1.727327,-0.684919,0.782007$

C, $-1.571878,-0.886702,-0.506901$

$\mathrm{H},-1.327005,-1.569996,-1.303068$

$\mathrm{H},-1.502777,-0.977013,1.796985$

C, $-2.486049,0.246243,-0.109646$

C, $0.549368,0.038028,-0.703166$

$\mathrm{H},-3.569845,0.124634,-0.202349$

$\mathrm{H},-2.150766,1.277800,-0.248887$

$\mathrm{Cl}, 1.665353,-1.101811,0.092296$

$\mathrm{Cl}, 0.685571,1.623498,0.094972$

Imag. freq. $=156.9$
$\mathbf{R}=\mathbf{C H}_{3}$

Abs. $\mathrm{E}=-1153.65399822$

C, $-2.008509,-0.277853,0.257919$

C, 1.215746, $-0.378462,0.699304$

C, $-1.667274,0.826238,-0.345992$

C, $-2.154259,-0.282161,-1.243703$

$\mathrm{H},-3.150110,-0.222580,-1.700593$

C, $-1.441401,-0.846164,-1.860504$

C, $-2.202892,-1.094789,1.476103$

C, $-1.119029,2.196998,-0.309973$

$\mathrm{Cl}, 1.427553,-1.544513,-0.603004$

$\mathrm{Cl}, 2.210076,1.037269,0.361670$

$\mathrm{H},-1.890979,-0.556122,2.376184$

$\mathrm{H},-1.622367,-2.023257,1.413053$

$\mathrm{H},-3.257720,-1.380187,1.582775$

$\mathrm{H},-0.770518,2.451020,0.697181$

$\mathrm{H},-0.262074,2.282477,-0.991381$

H, $-1.827213,2.978144,-0.615981$

Imag. freq. $=21.9$

\section{$\mathbf{R}=\mathbf{C H}=\mathbf{C H}_{2}$}

Abs. $\mathrm{E}=-1229.8204435$

C, $-1.075198,0.683109,-0.686259$

C, $0.207813,-1.302386,-0.562268$

C, $-0.140016,1.374167,-0.032569$

C, $-0.153089,1.536141,-1.522253$

$\mathrm{H},-0.537054,2.472687,-1.940050$

$\mathrm{H}, 0.613526,1.061963,-2.139976$

C, $-2.374344,0.048241,-0.711366$

C, $0.520581,1.758716,1.170554$ 
Cl, $-0.011462,-1.900216,1.107045$ $\mathrm{Cl}, 1.956557,-1.045657,-0.833673$ H, $-2.670102,-0.384613,-1.663841$ C, $-3.193594,-0.014893,0.347075$

$\mathrm{H},-4.164430,-0.496712,0.279813$

H, $-2.921589,0.413870,1.307521$

$\mathrm{H}, 0.128512,1.362267,2.105375$

C, 1.603547, 2.559367, 1.173534

H, 2.101624, 2.837058, 2.097091

$\mathrm{H}, 2.008683$, 2.958540, 0.248040

Imag. freq. $=135.0$

\section{Bicyclobutane 4}

\section{$\mathbf{R}=\mathbf{H}$}

Abs. $\mathrm{E}=-1075.13610689$

C, 0.981308, -0.797094, 0.761978

C, 0.981312, $-0.797097,-0.761970$

C, $-0.055697,-0.080299,0.000000$

C, 2.185777, $-0.338104,0.000007$

$\mathrm{H}, 3.036072,-1.018453,0.000010$

$\mathrm{H}, 2.453237,0.718387,0.000005$

Cl, $-0.186831,1.733327,-0.000004$

$\mathrm{Cl},-1.681255,-0.817428,-0.000003$

$\mathrm{H}, 0.855982,-1.597326,-1.478292$

H, 0.855974, -1.597321, 1.478303

\section{$\mathbf{R}=\mathbf{C H}=\mathbf{C H}_{2}$}

Abs. $\mathrm{E}=-1229.9337014$

C, $0.789950,-0.528256,-0.706789$

C, $-0.789408,-0.528679,-0.706972$

C, $-0.000105,0.526427,-0.038725$

C, $0.000428,-0.566190,-1.981773$

H, $0.000730,-1.519839,-2.512297$

H, $0.000268,0.307070,-2.635223$

Cl, $-0.000550,2.258179,-0.673594$

$\mathrm{Cl},-0.000539,0.571214,1.743981$

C, $-1.838237,-1.440377,-0.257256$

$\mathrm{H},-1.851569,-2.402326,-0.769585$

C, $-2.768298,-1.167037,0.663798$

$\mathrm{H},-3.523137,-1.902368,0.925155$

$\mathrm{H},-2.809465,-0.213605,1.179864$

C, $1.839174,-1.439406,-0.256857$

$\mathrm{H}, 1.853111,-2.401340,-0.769196$

C, 2.768964, $-1.165587,0.664331$

$\mathrm{H}, 3.524153,-1.900527,0.925780$

H, 2.809600, $-0.212123,1.180372$

\section{$\mathbf{R}=\mathbf{C H}_{\mathbf{3}}$}

Abs. $\mathrm{E}=-1153.77936312$

C, $0.835464,-0.358480,-0.766584$

C, $0.835416,-0.358345,0.766696$

C, $-0.377341,-0.015862,-0.000012$

C, $1.192895,-1.597595,0.000176$

$\mathrm{H}, 2.253197,-1.855707,0.000235$

$\mathrm{H}, 0.522761,-2.458385,0.000230$

$\mathrm{Cl},-1.883416,-1.067103,0.000035$

$\mathrm{Cl},-0.869122,1.704205,-0.000173$

C, $1.553713,0.355186,1.867784$

H, $1.279449,-0.064408,2.843055$

H, 2.638170, 0.245934, 1.752318

$\mathrm{H}, 1.308835,1.421444,1.875212$

C, $1.553832,0.354856,-1.867752$

$\mathrm{H}, 2.638282,0.245635,-1.752190$

$\mathrm{H}, 1.279639,-0.064918,-2.842965$

H, 1.308946, 1.421110, -1.875391

\section{$\mathbf{R}=\mathbf{P h}$}

Abs. $\mathrm{E}=-1537.24966242$

C, $0.794824,0.611531-0.627528$

C, $-0.743659,0.548006,-0.752420$

C, $-0.085405,1.578274,0.076792$

C, $0.131856,0.717805,-1.967195$

H, $0.227938,-0.158887,-2.606567$

$\mathrm{H}, 0.117689,1.668560,-2.500854$

$\mathrm{Cl},-0.122368,3.358513,-0.383662$

$\mathrm{Cl},-0.217886,1.448459,1.848075$

C, $-1.812734,-0.422856,-0.451770$

C, $-1.727155,-1.749929,-0.901732$

C, $-2.752579,-2.653854,-0.632434$

C, $-3.878186,-2.246638,0.087906$

C, $-3.970890,-0.928697,0.536955$

C, $-2.946345,-0.020779,0.269502$

H, $-0.852107,-2.077212,-1.457016$

$\mathrm{H},-2.671314,-3.678872,-0.984029$

$\mathrm{H},-4.677501,-2.952534,0.296124$

H, $-4.843909,-0.602459,1.095615$

H, $-3.024710,1.005242,0.615215$

C, $1.891829,-0.281712,-0.202171$

C, $1.791341,-1.133860,0.909104$ 
C, $2.857858,-1.961570,1.258218$

C, $4.033965,-1.954181,0.505765$

C, $4.139499,-1.115644,-0.605444$

C, $3.075200,-0.287419,-0.957860$

$\mathrm{H}, 0.876925,-1.155097,1.491341$

H, 2.765979, -2.618644, 2.118791

$\mathrm{H}, 4.862062,-2.601458,0.781599$

H, 5.049938, -1.105826, -1.198558

H, 3.156818, 0.367801, -1.820804

\section{CCA transition state $\mathbf{5}$}

\section{$\mathbf{R}=\mathbf{H}$}

Abs. $\mathrm{E}=-1075.07899701$

C, $0.025348,1.543755,-0.707817$

C, $-0.418216,0.393587,-0.044040$

C, $0.469118,0.856742,0.929330$

C, 1.374770, 1.589084, -0.027101

$\mathrm{H}, 2.122584,0.925490,-0.471239$

$\mathrm{H}, 1.768388,2.55983,0.283403$

H, - $0.513974,2.280575,-1.295247$

$\mathrm{H}, 0.303517,1.010947,1.992671$

Cl, $-1.938114,-0.366527,0.027266$

C, 1.209488, $-1.579112,-0.110314$

Imag. freq. $=652.8$

\section{$\mathbf{R}=\mathbf{C H}=\mathbf{C H}_{2}$}

Abs. $\mathrm{E}=-1229.90753434$

C, $0.900458,-0.672608,-0.646117$

C, $-0.055822,-0.043075,0.177715$

C, $-1.065560,-0.517465,-0.687383$

C, $-0.069099,-0.732990,-1.819130$

$\mathrm{H},-0.128378,-1.676768,-2.374891$

$\mathrm{H}, 0.022619,0.132946,-2.484587$

C, $2.184794,-1.266312,-0.463789$

C, $-2.444069,-0.861048,-0.574546$

$\mathrm{Cl},-0.079399,0.208168,1.887013$

$\mathrm{Cl}, 0.428433,2.280114,-0.661694$

$\mathrm{H}, 2.563254,-1.853531,-1.297629$

C, 2.947668, -1.095246, 0.638635

H, 3.934447, $-1.543211,0.704762$

H, 2.621567, $-0.486650,1.474910$

$\mathrm{H},-2.880642,-1.357795,-1.438702$

C, $-3.22343,-0.579248,0.494663$

H, $-4.276087,-0.84438,0.497328$

$\mathrm{H},-2.839992,-0.063452,1.368100$

Imag. freq. $=245.6$

\section{$\mathbf{R}=\mathbf{C H}_{\mathbf{3}}$}

Abs. $\mathrm{E}=-1153.7395141$

C, $1.253358,-0.302291,-0.565763$

C, $0.186885,0.303138,0.110363$

C, $0.004388,-0.956208,0.686998$

C, $0.647363,-1.689774,-0.471736$

H, $-0.070489,-1.917980,-1.267655$

H, 1.313891, $-2.526430,-0.232934$

C, $2.571994,0.177127,-1.038535$

C, $-0.360981,-1.401226,2.046737$

$\mathrm{Cl}, 0.013785,1.885389,0.742571$

$\mathrm{Cl},-2.034930,-0.039031,-1.136946$

$\mathrm{H}, 2.779746,1.194067,-0.695481$

$\mathrm{H}, 2.582181,0.172108,-2.138803$

H, 3.376256, $-0.492627,-0.711034$

H, $-1.352774,-1.869610,1.976994$

$\mathrm{H},-0.421431,-0.567741,2.750715$

$\mathrm{H}, 0.334037,-2.164482,2.414182$

Imag. freq. $=388.1$

\section{$\mathbf{R}=\mathbf{P h}$}

Abs. $E=-1537.2305944$

C, $-0.991526,-0.157931,0.387965$

C, $-0.005244,0.784059,0.016827$

C, $0.976046,-0.163782,0.382886$

C, $-0.008141,-0.919276,1.264196$

$\mathrm{H},-0.011664,-2.013609,1.191335$

$\mathrm{H},-0.001494,-0.573848,2.305059$

C, $-2.336156,-0.461444,0.012143$

C, $2.316522,-0.478277,0.001700$

$\mathrm{Cl},-0.006395,1.954379,-1.263954$

$\mathrm{Cl}, 0.074601,2.276856,1.997437$

C, $-2.977035,-1.578925,0.598471$

C, $-3.057348,0.328350,-0.915859$

C, $-4.286135,-1.89895,0.263484$

C, $-4.367272,0.002155,-1.239923$

C, $-4.984273,-1.110837,-0.657141$

H, $-2.439645,-2.180445,1.324962$

$\mathrm{H},-2.585718,1.195167,-1.362713$

$\mathrm{H},-4.768467,-2.756731,0.722994$

H, $-4.914821,0.617667,-1.947639$

H, $-6.009913,-1.359144,-0.915409$

C, 2.943699, -1.614329, 0.565864

C, 3.046759, 0.323119, -0.909115 
C, 4.249326, -1.942018, 0.224770

C, $4.353498,-0.010174,-1.238445$

C, $4.956819,-1.142304,-0.678551$

H, 2.398936, $-2.224825,1.279145$

H, 2.584975, 1.205038, -1.336250

$\mathrm{H}, 4.721832,-2.814579,0.666226$

$\mathrm{H}, 4.909238,0.614300,-1.931704$

H, 5.979993, -1.396571, -0.940844

Imag. freq. $=177.6$

\section{Cyclobutene 6}

\section{$\mathbf{R}=\mathbf{H}$}

Abs. $\mathrm{E}=-1075.17128848$

C, $-0.539063,1.65884,-0.350721$

C, $-0.694302,0.384425,0.029086$

C, $0.699227,0.225499,0.570626$

C, $0.926358,1.689506,0.058773$

$\mathrm{H}, 1.642543,1.748988,-0.765753$

H, 1.201344, 2.408865, 0.838984

H, - $1.206907,2.408883,-0.758595$

$\mathrm{H}, 0.777772,0.090015,1.650578$

Cl, $-2.013131,-0.728422,0.012743$

$\mathrm{Cl}, 1.732656,-1.060192,-0.178142$

\section{$\mathbf{R}=\mathbf{C H}=\mathbf{C H}_{2}$}

Abs. $\mathrm{E}=-1229.97177299$

C, $-1.304441,-0.249671,0.042399$

C, $-0.310329,0.642717,-0.147317$

C, $0.819700,-0.328713,0.050523$

C, $-0.315603,-1.381987,0.312200$

$\mathrm{H},-0.317236,-1.774896,1.332131$

$\mathrm{H},-0.346880,-2.207156,-0.406165$

Cl, $-0.271551,2.325864,-0.532031$

$\mathrm{Cl}, 1.810975,0.050704,1.578607$

C, $-2.744731,-0.203441,-0.012075$

$\mathrm{H},-3.187714,0.761962,-0.250996$

C, $-3.531434,-1.267338,0.213029$

$\mathrm{H},-4.613160,-1.193644,0.162677$

$\mathrm{H},-3.118432,-2.243162,0.455922$

C, $1.782661,-0.512924,-1.078812$

H, 2.277180, 0.402414, -1.399850

C, 2.062709, -1.669229, - 1.680607

H, 1.611743, $-2.610841,-1.380508$

H, 2.773098, -1.712817, - 2.501044

\section{$\mathbf{R}=\mathbf{C H}_{\mathbf{3}}$}

Abs. $\mathrm{E}=-1153.81507332$

C, $1.229843,-0.881777,-0.074611$

C, $0.559880,0.273310,0.051864$

C, $-0.781859,-0.339422,0.344549$

C, $-0.035595,-1.707638,0.141527$

$\mathrm{H},-0.383495,-2.273569,-0.727771$

H, $-0.037756,-2.356315,1.026928$

$\mathrm{Cl}, 1.029315,1.940206,-0.013074$

$\mathrm{Cl},-2.040494,-0.009137,-0.958962$

C, $-1.409557,-0.073400,1.702513$

$\mathrm{H},-2.294818,-0.699130,1.849128$

$\mathrm{H},-0.682344,-0.297771,2.491772$

$\mathrm{H},-1.705885,0.975749,1.792267$

C, $2.644753,-1.273845,-0.302348$

$\mathrm{H}, 3.018599,-1.892529,0.524154$

H, 2.741222, $-1.873037,-1.217268$

H, 3.289726, -0.394936, -0.395561

\section{$\mathbf{R}=\mathbf{P h}$}

Abs. $\mathrm{E}=-1537.28649668$

C, $1.060197,0.103110,-0.333383$

C, $0.135667,0.792169,0.369683$

C, $-1.013827,0.553133,-0.567213$

C, $0.053366,-0.223241,-1.433431$

$\mathrm{H}, 0.250607,0.247827,-2.400466$

$\mathrm{H},-0.179508,-1.284785,-1.569721$

$\mathrm{Cl}, 0.169768,1.704531,1.841862$

C, $2.461850,-0.259477,-0.206059$

C, 3.239026, 0.128622, 0.903288

C, $4.578554,-0.239270,0.987035$

C, 5.168376, $-0.999128,-0.027451$

C, 4.408533, -1.390087, -1.131535

C, 3.067750, -1.023542, -1.220446

H, 2.789167, 0.718610, 1.694442

H, 5.166441, 0.067887, 1.847649

H, 6.214608, $-1.283742,0.042944$

H, 4.860529, -1.979814, -1.924269

H, 2.480636, -1.328174, -2.082509

$\mathrm{Cl},-1.553757,2.120444,-1.393686$

C, $-2.215899,-0.243909,-0.106502$

C, $-2.198575,-0.892616,1.135088$

C, $-3.268681,-1.69771,1.531249$

C, $-4.368310,-1.868417,0.691875$ 
C, $-4.391744,-1.227765,-0.549238$

C, $-3.326130,-0.422454,-0.945054$

$\mathrm{H},-1.349664,-0.769989,1.799317$

$\mathrm{H},-3.239905,-2.186544,2.501324$

$\mathrm{H},-5.202575,-2.491886,1.001755$

$\mathrm{H},-5.244459,-1.352021,-1.211328$

H, $-3.358988,0.081604,-1.905567$

Transition state connecting bicyclobutane $\mathbf{4}$ to butadiene 3

\section{$\mathbf{R}=\mathbf{H}$}

Abs. $\mathrm{E}=-1075.0589605$

C, $1.010860,-1.030049,-0.347201$

C, $-0.107437,-0.088546,0.247175$

C, $0.652435,-0.795392,1.188454$

C, $2.262516,-0.460790,-0.476932$

$\mathrm{H}, 3.113166,-1.043438,-0.822222$

$\mathrm{H}, 2.445530,0.557158,-0.155668$

$\mathrm{H}, 0.714689,-1.950226,-0.843340$

$\mathrm{H}, 1.358394,-0.355148,1.886202$

Cl, $-0.056596,1.711223,-0.049931$

$\mathrm{Cl},-1.739994,-0.708851,-0.169714$

Imag. freq. $=198.2$
$\mathbf{R}=\mathbf{C H}_{\mathbf{3}}$

Abs. $\mathrm{E}=-1153.69279365$

C, $1.122943,-0.437310,-0.422768$

C, $-0.167537,0.215275,0.136895$

C, $0.079669,-0.644700,1.224251$

C, $0.986935,-1.626023,-1.053592$

$\mathrm{H}, 1.862877,-2.159939,-1.413555$

$\mathrm{H}, 0.020767,-2.083923,-1.223592$

C, $2.476915,0.211128,-0.233358$

C, $-0.761974-1.816539,1.559786$

Cl, $-0.061204,1.984894,0.466200$

$\mathrm{Cl},-1.689513,0.013461,-1.008054$

$\mathrm{H}, 2.392562,1.230316,0.141991$

$\mathrm{H}, 3.009751,0.240674,-1.189994$

$\mathrm{H}, 3.075910,-0.362628,0.480306$

$\mathrm{H},-1.389543,-1.428732,2.381533$

$\mathrm{H},-1.447536,-2.176913,0.780967$

$\mathrm{H},-0.184305,-2.641859,1.986586$

Imag. freq. $=167.4$

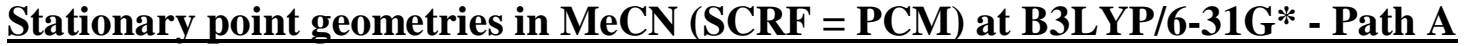

Cyclopropene $\mathbf{1}+: \mathrm{CCl}_{2}$

$\mathbf{R}=\mathbf{H}(r=3.1 \AA)$

Abs. $\mathrm{E}=-1075.0058416$

C, $2.067105,-1.066269,0.342657$

C, $1.997915,-0.423547,0.784788$

$\mathrm{H}, 1.777185,-0.414103,1.840953$

H, .919314, $-1.974308,-0.909418$

C, 2.558880, 0.353801, -0.378905

C, $-0.907823,0.053005,0.793582$

$\mathrm{H}, 1.952581,1.130495,-0.858639$

H, 3.632668, 0.556891, -0.432042

$\mathrm{Cl},-1.779249,-1.129015,-0.174525$

$\mathrm{Cl},-0.784175,1.552490,-0.106751$
$\mathbf{R}=\mathbf{C H}_{\mathbf{3}}(r=3.5 \AA)$

Abs. $\mathrm{E}=-1153.65429832$

C, $-1.822804,0.634471,-0.429618$

C, $-2.025803,-0.433895,0.287012$

C, $-2.261302,-1.111389,1.580935$

C, $-1.641799,2.095198,-0.572024$

C, $-1.952590,-0.653279,-1.203303$

C, $1.442672,-0.380094,0.754959$

$\mathrm{H},-1.065207,-1.123466,-1.648169$

$\mathrm{H},-2.866522,-0.861792,-1.773168$

$\mathrm{Cl}, 1.760635,-1.376884,-0.647795$

$\mathrm{Cl}, 2.077992,1.231511,0.472662$

$\mathrm{H},-1.490499,-1.868537,1.770276$

H, $-3.227847,-1.631919,1.573246$

H, $-2.257118,-0.401781,2.415070$

$\mathrm{H},-0.682504,2.327021,-1.054071$

$\mathrm{H},-1.670817,2.604307,0.397180$

$\mathrm{H},-2.426387,2.521433,-1.210863$

$\mathbf{R}=\mathbf{C H}=\mathbf{C H}_{2}(r=3.4 \AA)$

Abs. $\mathrm{E}=-1229.82582993$

C, $-1.639348,-0.587668,0.060754$ 
C, $-1.720090,0.655321,0.497928$

C, $-1.726421,2.077380,0.282018$

C, $-1.476466,-1.543088,-0.993898$

C, $-1.904880,-0.424509,1.535648$

C, 1.673612, 0.144129, 0.829801

$\mathrm{H},-2.906990,-0.633793,1.928293$

H, $-1.106503,-0.624400,2.257455$

$\mathrm{Cl}, 2.135770,1.415395,-0.281712$

$\mathrm{Cl}, 2.106661,-1.410835,0.153667$

H, $-1.890544,2.697489,1.164280$

C, $-1.540315,2.662898,-0.915101$

$\mathrm{H},-1.552238,3.744512,-1.019899$

$\mathrm{H},-1.373448,2.080895,-1.818776$

$\mathrm{H},-1.332244,-1.150741,-2.002335$

C, $-1.492493,-2.872651,-0.786478$

$\mathrm{H},-1.634154,-3.286757,0.209105$

$\mathrm{H},-1.366802,-3.575589,-1.605383$

Cyclopropene $\mathbf{1}+: \mathrm{CCl}_{2}-$ Transition state for stepwise addition

$\mathbf{R}=\mathbf{H}(r=2.5 \AA)$

Abs. $\mathrm{E}=-1075.00552351$

C, $-1.695433,-0.735375,0.758158$

C, $-1.562387,-0.869464,-0.543065$

$\mathrm{H},-1.347196,-1.521301,-1.374115$

H, $-1.470995,-1.095219,1.752198$

C, $-2.468525,0.239644,-0.068407$

C, 0.568582, 0.048891, -0.726901

$\mathrm{H},-2.137522,1.278788,-0.153040$

$\mathrm{H},-3.553140,0.120781,-0.146654$

$\mathrm{Cl}, 1.666113,-1.088559,0.101094$

$\mathrm{Cl}, 0.654795,1.624722,0.099077$

Imag. freq. $=166.2$

$\mathbf{R}=\mathbf{C H}=\mathbf{C H}_{2}(r=2.5 \AA)$

Abs. $\mathrm{E}=-1229.82635274$

C, $-0.146067,1.359926,-0.009934$

C, $-1.103256,0.709000,-0.675977$

C, $-2.410456,0.092935,-0.704186$

C, $0.523623,1.712499,1.198097$

C, $-0.175229,1.572751,-1.493675$

C, $0.223779,-1.302230,-0.607559$

$\mathrm{H},-0.544939,2.532450,-1.870617$

$\mathrm{H}, 0.577770,1.110128,-2.137228$

Cl, $-0.013530,-1.928617,1.052628$

$\mathrm{Cl}, 1.978681,-0.994459,-0.830654$

$\mathrm{H},-2.753824,-0.253723,-1.678889$

C, $-3.190087,-0.045747,0.378814$

$\mathrm{H},-4.172342,-0.505865,0.308260$

$\mathrm{H},-2.876202,0.301225,1.360450$

$\mathrm{H}, 0.124187,1.317761,2.133229$

C, 1.623604, 2.491680, 1.199797

H, 2.030837, 2.892902, 0.274722

$\mathrm{H}, 2.131479,2.752522,2.124257$

Imag. freq. $=126.3$

\section{$\mathbf{R}=\mathbf{C H}_{3}$}

All structures with $r<3.5 \AA$ were lower in energy than the starting structure $(r=3.5 \AA)$. 


\section{1,3-Dihalobutadiene 3}

$\mathbf{R}=\mathbf{H}$

Abs. $\mathrm{E}=-1075.18070813$

C, $-2.051720,0.128771,0.000470$

C, $-0.810263,-0.622712,0.000819$

$\mathrm{H},-0.882982,-1.708163,0.000789$

$\mathrm{H},-1.983845,1.213809,0.000358$

C, $-3.254485,-0.464751,0.000121$

C, $0.427759,-0.105552,0.000495$

$\mathrm{H},-3.360292,-1.547246,0.000247$

H, $-4.171969,0.115744,0.000036$

$\mathrm{Cl}, 1.849824,-1.114212,-0.000447$

$\mathrm{Cl}, 0.769666,1.603113,-0.000310$

\section{$\mathbf{R}=\mathbf{C H}=\mathbf{C H}_{2}$}

Abs. $\mathrm{E}=-1229.96361829$

C, $1.465319,0.120650,0.360688$

C, $0.040759,0.526913,0.075464$

C, $1.943632,0.176005,1.612205$

C, $-0.922597,-0.408488,-0.006710$

$\mathrm{H}, 1.327400,0.509301,2.443136$

H, 2.962941, $-0.125475,1.840120$

$\mathrm{Cl},-2.587776,-0.101409,-0.441706$

$\mathrm{Cl},-0.611926,-2.110323,0.276101$

C, $-0.139123,1.973554,-0.163173$

C, 2.271593, $-0.270628,-0.816340$

$\mathrm{H}, 0.701102,2.437327,-0.683888$

C, $-1.144684,2.751146,0.256051$

$\mathrm{H},-1.136442,3.818117,0.047967$

$\mathrm{H},-1.986769,2.370023,0.825405$

H, 1.730787, -0.786621, -1.612191

C, 3.574954, $-0.007799,-0.976111$

H, 4.111201, -0.341636, -1.860829

H, 4.145600, 0.550302, -0.236885
$\mathbf{R}=\mathbf{C H}_{\mathbf{3}}$

Abs. $\mathrm{E}=-1153.80529188$

C, $-1.775305,0.052064,0.097946$

C, $-0.380841,0.641789,0.044582$

C, $-0.370237,2.151731,0.003284$

C, $-2.412012,-0.259348,-1.231212$

C, $-2.386289,-0.115043,1.271252$

C, $0.772644,-0.079531,0.016678$

$\mathrm{H},-1.895627,0.110378,2.214304$

H, $-3.406123,-0.489952,1.326738$

$\mathrm{Cl}, 2.371716,0.616388,-0.053193$

$\mathrm{Cl}, 0.775753,-1.827002,0.053110$

$\mathrm{H}, 0.615819,2.570592,-0.204183$

$\mathrm{H},-0.724293,2.551439,0.962283$

H, $-1.067773,2.510724,-0.763418$

H, $-3.443595,-0.602288,-1.103458$

$\mathrm{H},-2.422397,0.620624,-1.887531$

H, -1.850739, -1.041056, - 1.758519

$\mathbf{R}=\mathbf{P h}$

Abs. $E=-1537.28715257$

C, $-0.685743,-0.442601,0.349710$

C, $0.619381,-0.815484,0.989970$

C, $1.866828,-0.438320,0.268791$

C, $-0.953781,1.024288,0.222884$

C, 0.627167, -1.415170, 2.191436

C, $-1.559559,-1.376087,-0.068501$

$\mathrm{H}, 1.556241,-1.688517,2.685499$

H, $-0.296051,-1.661922,2.708344$

$\mathrm{Cl},-1.242271,-3.099160,-0.048982$

$\mathrm{Cl},-3.155261,-1.012416,-0.694444$

C, $1.921196,-0.473109,-1.135107$

C, 3.022788, $-0.056519,0.972356$

C, 3.099539, $-0.158207,-1.812944$

C, 4.199944, 0.259111, 0.294941

C, $4.244339,0.207618,-1.101033$

$\mathrm{H}, 1.039890,-0.764385,-1.702623$

H, 2.992977, 0.014787, 2.057854

H, 3.122352, - $0.199849,-2.901267$

H, 5.082571, 0.557676, 0.859422

H, 5.162568, 0.458792, - 1.630318

C, $-1.238012,1.614790,-1.018213$

C, $-0.869742,1.842067,1.360897$

C, $-1.447567,2.990709,-1.115475$

C, $-1.091274,3.216224,1.263441$

C, $-1.379576,3.794803,0.025012$

H, $-1.283944,0.996869,-1.912530$

H, $-0.634361,1.398718,2.326364$

H, - $1.660739,3.435289,-2.086780$

$\mathrm{H},-1.033767,3.836007,2.157348$

$\mathrm{H},-1.545266,4.868640,-0.052053$ 